\title{
EVALUACIÓN BIOÉTICA DE TRABAJOS DE INVESTIGACIÓN EN SERES HUMANOS PUBLICADOS EN AMÉRICA LATINA Y EL CARIBE
}

\author{
Roberto Mancini Rueda." \\ Fernando Lolas Stepke**
}

\section{Resumen}

La mención explícita de requisitos bioéticos en publicaciones biomédicas que involucran seres humanos y la incorporación de criterios éticos en la evaluación de trabajos científicos fueron investigadas en una muestra de revistas de la Región de América Latina y el Caribe. Mediante un criterio de selección basado en indexación en bases MEDLINE y LILACS, obtención de texto completo y de instrucciones a los autores, entre otros, se identificó 41 revistas científicas, siendo revisados 625 artículos. Los resultados varían según se trate de ensayos con medicamentos (177 artículos) o estudios clínicos de diagnóstico o tratamiento no farmacológico (448), existiendo mayor preocupación de los investigadores en el primer caso ( $49 \%$ señala consentimiento informado y $46 \%$ revisión por comité de ética) que en el segundo (32\% indica obtener consentimiento y $27 \%$ revisión por comité). Además, se aprecia mayor cumplimiento de disposiciones éticas en los ensayos clínicos fase II y III que en los fase IV. El análisis de las instrucciones a los autores, demuestra que en las revistas revisadas la mayor preocupación de los editores se refiere al consentimiento informado en las revistas indexadas

Médico Cirujano, Consultor Programa Regional de Bioética OPS/OMS.

Correspondencia : mancinir@chi.ops-oms.org

** Profesor Titular, Facultad de Medicina de la Universidad de Chile. Director Programa Regional de Bioética OPS/OMS

Correspondencia: lolasf@chi.ops-oms.org

\begin{abstract}
Biothical evaluation of research involving human subjects published in Latin America and the Caribbean
\end{abstract}

The explicit mention of bioethical requirements in biomedical publications involving human subjects and the inclusion of ethical criteria in the evaluation of scientific papers were investigated in a sample of journals from the Latin American and Caribbean Region. Through a selection criterion based on the indexation in databases MEDLINE and LILACS, access to complete texts and guidelines for contributors, among others, 41 scientific journals were identified, out of which 625 articles were reviewed. The results differ for drug trials (177 articles) or diagnostic clinical studies or non pharmacological treatment (448). A greater concern for ethical aspects was evident among the former (49\% indicate informed consent and $46 \%$ review by ethics committee) than in the latter (32\% indicate obtaining consent and $27 \%$ review by ethics committee). Also, a greater compliance with ethical regulations is observed in clinical trials phase II and III than in phase IV. The analysis of guidelines for constributors shows that in the reviewed journals editors' greatest concern refers to informed consent in the journals indexed in MEDLINE (50\% asks for this requirement) and the approval by the ethics committees in the ones indexed in LILACS (in $43 \%$ it is requested). In general, the explicit compliance with ethical requirements reaches at the most $50 \%$ and there 
Evaluación bioética de trabajos de investigación en seres humanos publicados en América Latina y el Caribe - R. Mancini y F. Lolas

en MEDLINE (50\% pide ese requisito) y la aprobación por comité de ética en las indexadas en LILACS (en $43 \%$ se solicita). En general, el cumplimiento explícito de requisitos éticos llega como máximo al 50\% y no hay referencias éticas en cerca del $45 \%$ de las publicaciones.

PALABRAS CLAVE: Consentimiento informado; comité de ética; investigación clínica; publicaciones. are no ethical references in almost $45 \%$ of the publications.

KEY WORDS: Informed consent, ethics committee, clinical research, publications. 


\section{Resuno}

Avaliação bioética dos trabalhos de investigação em seres humanos publicados na América Latina e Caribe

A menção explícita de requisitos bioéticos nas publicações que envolvem seres humanos e a incorporação de critérios éticos na avaliação de trabalhos científicos foram estudadas em a mostra expressiva de revistas da América Latina e Caribe.

Mediante um critério de seleção baseado em indexação nas bases MEDLINE e LILACS, a presença de texto completo de instruções aos autores foram analisadas 41 revistas científicas e 625 artigos.

Os resultados variaram segundo a característica da publicação: ensaios com medicamentos (177 artigos) ou estudos clínicos de diagnóstico ou tratamento não farmacológico (448), existindo maior preocupação dos investigadores no primeiro caso ( $49 \%$ contendo termo de consentimento informado e $46 \%$ revisão por Comitê de Ética) que no segundo grupo (32\% tendo obtido termo de consentimento informado e $27 \%$ com revisão por Comitê de Ética).

Foi observado, também, maior cumprimento de disposições éticas nos ensaios clínicos em fase II e III que na fase IV.

Com relação a análise das instruções aos autores, ficou demonstrado que houve maior preocupação dos editores relativa ao consentimento informado nas revistas indexadas no MEDLINE (50\% exige esse requisito) e aprovação por Comitê de Ética nas indexadas na base LILACS (43\%).

Em geral o cumprimento explícito de requisitos éticos atingem o máximo de $50 \%$ e não se obteve requisitos éticos em cerca de 45\% das publicações.

PALAVRAS CHAVES: Consentimento Informado; comitê de ética; investigação clínica; publicações.

\section{Résumé}

Évaluation bioéthique de travaux de recherche effectués sur des humains et publiés en Amérique Latine et aux Caraïbes.

À partir d'un échantillon de revues publiées dans la zone d'Amérique Latine et des Caraïbes, on a recherché dans les publications médicales la référence explicite des conditions bioéthiques impliquant des humains et l'incorporation de critères éthiques sur l'évaluation de travaux scientifiques. Au moyen d'un critère de sélection basé sur l'ndexation dans les bases MEDLINE et LILACS, on a obtenu un texte complet et des consignes [adressées] aux auteurs, entre autres ; on a identifié 41 revues scientifiques et 625 articles ont été analysés. Les résultats varient selon qu'il s'agit d'essais avec des médicaments (177 articles) ou d'études cliniques de diagnostique ou de traitement non pharmacologique (448 articles). Il existe un plus grand souci des chercheurs dans le premier cas $(49 \%$ signalent le consentement informé et $46 \%$ signalent la révision du comité d'éthique) par rapport au deuxième cas $(32 \%$ indiquent l'obtention du consentement et $27 \%$ la révision du comité). Par ailleurs, on constate une meilleure application des dispositions éthiques dans les essais cliniques phase II et III que dans la phase IV. L'analyse des consignes données aux auteurs démontre que dans les revues analysées, le souci mayeur des éditeurs concerne le consentement informé dans les revues indexées dans MEDLINE (50\% exige cette condition) et l'approbation du comité d'éthique dans celles indexées dans LILACS (sollicitée dans $43 \%$ des cas). En général, l'application explicite des exigences éthiques atteint un pourcentage maximum de $50 \%$ et dans à peu près $45 \%$ des publications, il n'existe pas de références éthiques.

MOTS CLÉS : Consentement informé; comité d'éthique; recherche clinique; publications. 
Evaluación bioética de trabajos de investigación en seres humanos publicados en América Latina y el Caribe - R. Mancini y F. Lolas

\section{Introducción}

Una investigación realizada en 1999 permitió concluir que existe poca preocupación en cuanto a supervisar el cumplimiento de estándares éticos y bioéticos básicos en los protocolos de investigación que utilizan sujetos humanos, por parte de los organismos nacionales de ciencias y tecnología (ONCYT) encargados de su aprobación ( 1 ) .

Habitualmente dicha evaluación es entregada a comités de ética institucionales (universitarios, hospitalarios u otros). No obstante, el mismo estudio anterior permitió observar que en el $48 \%$ de los países analizados no existían normativas ni comités de evaluación ética-científica en las instituciones que financian investigación biomédica.

El exiguo desarrollo de la bioética en el campo de la investigación con seres humanos parece ser un reflejo de la escasa investigación biomédica en general en América Latina y el Caribe, tanto en cantidad como en calidad. Como lo demuestran algunos estudios recientes, en cinco países (México, Brasil, Argentina, Chile y Venezuela) se concentra cerca del $80 \%$ del total de proyectos de la Región, con una producción de publicaciones científicas que en un $50 \%$ se circunscribe sólo al medio nacional ( 2,3 ).

No obstante, aunque cabe suponer que también es insuficiente, se carece de una información objetiva respecto de la revisión que se está haciendo - desde una perspectiva ética- de los protocolos de investigación que son presentados para publicación en revistas, cuando se incluye a seres humanos como sujetos de estudio.

Tiende a considerarse como una medida de "calidad" de una revista científica su indización en la base de datos denominada
MEDLINE $^{\mathrm{i}}$. Del total de revistas existentes en la base latinoamericana llamada LILACS ${ }^{\mathrm{ii}}$ (cerca de 600) 47 están incorporadas a la base MEDLINE. No obstante, se desconoce la consideración de aspectos éticos en esa selección como estándar de calidad.

El propósito de este estudio es, en primer lugar, determinar el cumplimiento explícito de requisitos bioéticos básicos por parte de los investigadores que realizan estudios científicos en seres humanos y, en segundo término, establecer si existen criterios éticos incorporados en la evaluación de los trabajos que son presentados para su publicación en una muestra representativa de las principales revistas científicas de la Región, indexadas en las bases bibliográficas LILACS y MEDLINE.

MEDLINE: Base de datos bibliográfica administrada por la NLM (United States National Library of Medicine), que corresponde al sistema "en línea" del Index Medicus.. Según la define su URL (http:// nlm.nih.gov/ pubs/factsheets/jsel.html) "es usada internacionalmente para proveer acceso a las revistas biomédicas del mundo". MEDLINE establece ciertos requisitos para la indexación, basados "tanto en políticas científicas como en calidad científica, para lo cual disponen de un Comité Técnico de Revisión y Selección de la Literatura que revisa periódicamente las revistas indexadas y establece la calidad de sus contenidos". Sin embargo, al revisar los requisitos exigidos para indizar una revista, no se encuentra una exigencia, por ejemplo, de cumplimiento de resguardos éticos en investigación con seres humanos (ver http:// nlm.nih.gov/pubs/ factsheets/jfaq.html)

ii LILACS (Literatura Latino-Americana y del Caribe en Ciencias de la Salud): Base de datos bibliográfica administrada por BIREME (Centro Latinoamericano y del Caribe para Información en Ciencias de la Salud) que contiene "toda la literatura relacionada con Ciencias de la Salud producida por autores latinoamericanos y del Caribe, publicada en los países de la Región desde 1982" (ver http://www.bireme.br). La base LILACS pretende incluir toda la literatura relacionada con Ciencias de la Salud que se produce en los países de América Latina y el Caribe, por lo que no hace una selección basada en criterios de calidad. 


\section{Material y método}

Este trabajo corresponde a un estudio descriptivo. Se revisó la existencia de contenidos que establezcan un respeto por los principios bioéticos en los trabajos de investigación en seres humanos, editados en dos grupos seleccionados de revistas científicas de la Región, de acuerdo a criterios establecidos en la muestra. Además se revisó las normas de publicación o instrucciones a los autores, respecto de la existencia o no de requisitos éticos. Con estos dos elementos se hizo una calificación cualitativa de las revistas desde una perspectiva ética y se comparó entre ambos grupos en aquellos aspectos que lo permitían.

\section{Características de la muestra}

- Revistas científicas indexadas en bases MEDLINE o LILACS;

- que hayan sido publicadas desde enero de 1997 hasta diciembre de 1998 (dos años en total);

- que contengan artículos de investigación en seres humanos, de los siguientes tipos:

- Ensayos clínicos con medicamentos (Fases I, II, III o IV) y otros productos biológicos (vacunas, productos naturales). Para efectos de este trabajo se han denominado "estudios farmacológicos". iii

- Estudios clínicos de diagnóstico o terapia no farmacológica, retrospectivos y prospectivos. Para efectos de este trabajo, se han denominado "estudios no

Estudios farmacológicos: Ensayos clínicos con medicamentos $\mathrm{u}$ otros productos de origen farmacológico o biológico. Implican cumplimiento de requisitos éticos tales como: consentimiento informado, evaluación por comité de ética, evaluación y cobertura de efectos adversos, privacidad y confidencialidad de los datos, conocimiento de resultados.

\section{farmacológicos". iv}

- que puedan ser obtenidas en texto completo para su revisión, y

- que hayan sido editadas en siete países seleccionados de América Latina y el Caribe, con mayor desarrollo tecno-científico, de acuerdo al volumen global de publicaciones existente en LILACS y MEDLINE: Argentina, Brasil, Chile, Cuba, México, Puerto Rico y Venezuela.

\section{Selección de la muestra}

De un total de 43 revistas editadas en los países preseleccionados, que estaban registradas en MEDLINE en diciembre de 1999 (http://www.ulm.nih.gov/tsd/serials/lji.htm), 28 contenían artículos de investigaciones en seres humanos y, de ellas, en 18 fue posible obtener los textos completos. Se seleccionó el $100 \%$ de las revistas, lo que constituye el grupo A del estudio (ver Anexo 1).

De acuerdo a la información obtenida en LILACS en diciembre de 1999 (http:// www.bireme.br) excluyendo las revistas indizadas también en MEDLINE (grupo A) existían otras 426 revistas editadas en los países preseleccionados. De ellas, 197 contenían artículos de investigaciones en seres humanos, y de éstas, en 46 era posible conseguir el texto completo. Por método aleatorio simple, se seleccionó el 50\% (23 revistas) para su revisión, lo que constituye el grupo B del estudio (ver Anexo 2).

iv Estudios no farmacológicos: Estudios clínicos de diagnóstico o tratamiento que no utilizan productos farmacéuticos o biológicos, pero sí técnicas "invasivas" (punciones, cateterizaciones, cirugía, etc.) o "intervencionistas" (modificación de conductas o de hábitos, conocimiento de información confidencial, cambios de características biológicas o psicológicas, etc.). Desde una perspectiva bioética, se supone que implican cumplimiento de requisitos éticos básicos similares a los estudios farmacológicos: consentimiento informado, evaluación por comité de ética, privacidad y resguardo de la identidad de los pacientes y los datos obtenidos, conocimiento de los resultados. 
Evaluación bioética de trabajos de investigación en seres humanos publicados en América Latina y el Caribe - R. Mancini y F. Lolas

\section{Resultados}

\section{Análisis de la muestra}

Grupo A: Revistas indexadas en LILACS y MEDLINE.

De un total de 2.974 artículos publicados en 1997 y 1998 en las 18 revistas seleccionadas, se encontró 434 referidos a investigaciones clínicas en seres humanos (Tabla 1). De las revistas revisadas, una sólo tiene artículos indizados en uno de los dos años considerados para el estudio.

Del total de artículos, 93 se refieren a investigaciones clínicas con medicamentos $\mathrm{u}$ otros productos farmacológicos o biológicos y 341 a investigaciones clínicas no farmacológicas, de acuerdo a las definiciones entregadas previamente.

TABLA 1: Distribución por países de revistas del grupo A (MEDLINE)

\begin{tabular}{lllll|}
\hline - Argentina: & 4 & $=93$ & artículos \\
- Brasil: & 5 & $=123$ & artículos \\
- Chile: & 2 & $=73$ & artículos \\
- Cuba: & 1 & $=8$ & artículos \\
- México & 4 & $=119$ & artículos \\
- Puerto Rico: & 1 & $=12$ & artículos \\
- Venezuela: & 1 & $=6$ & artículos \\
Total & 18 & $=434$ & artículos \\
\hline
\end{tabular}

Grupo B: Revistas indexadas sólo en LILACS.

De un total de 2.354 artículos publicados en 1997 y 1998 en las 23 revistas seleccionadas, se encontró 191 que se refieren a investigaciones en seres humanos (Tabla 2). De las revistas revisadas, una sólo tiene artículos indizados en uno de los dos años considerados para el estudio.

Del total de artículos, 84 se refieren a investigaciones clínicas con medicamentos u otros productos farmacológicos o biológicos y 107 a investigaciones clínicas no farmacológicas, de acuerdo a las definiciones entregadas previamente.

Las temáticas abordadas resultaron bastante amplias, por lo que -aunque no era un objetivo de la selección- permitió analizar el componente ético utilizado en diferentes especialidades de la Medicina.

TABLA 2: Distribución por países de revistas del grupo B (LILACS)

\begin{tabular}{lll}
\hline - Argentina & $5=36$ artículos \\
- Brasil: & $7=86$ artículos \\
- Chile: & $4=21$ artículos \\
- Cuba: & $2=9$ artículos \\
- México & $4=33$ artículos \\
- Venezuela: & $1=6$ artículos \\
Total & $23=191$ artículos \\
\hline
\end{tabular}

\section{Análisis de los artículos publicados y contenidos éticos}

En general, las revistas indizadas en MEDLINE publican un porcentaje mayor de artículos referidos a investigaciones clínicas que las revistas registradas sólo en LILACS (434 de $2.974=14,6 \%$ v/s 191 de $2.354=$ $8,1 \%$ ), debido a un mayor número de artículos sobre investigación en diagnóstico y terapia no farmacológica (341 en MEDLINE v/s 107 en LILACS).

Porcentualmente, el número de artículos referidos a investigación con medicamentos es similar en las revistas indexadas en LILACS (84 de $2.354=3,6 \%)$ y en MEDLINE (93 de $2.974=3,1 \%$ )

Las revistas indizadas en LILACS publican en mayor proporción artículos de revisión de literatura o de análisis de casos con una opinión teórica. 
TABLA 3: Mención de requerimientos éticos en la investigación realizada en artículos de revistas indexadas en MEDLINE $(\mathrm{N}=18)$

\begin{tabular}{|lrr|}
\hline & Est.Farmacológicos & Est. No farmacológicos \\
Consentimiento informado & $(\mathrm{N}=93)$ & $(\mathrm{N}=341)$ \\
Privacidad de datos & $47(50,5 \%)$ & $109(32,0 \%)$ \\
Seguro efectos adversos & $6(6,5 \%)$ & $15(4,4 \%)$ \\
Revisión por Comité de Ética & $9(9,7 \%)$ & $6(1,8 \%)$ \\
Sin referencias éticas & $39(41,9 \%)$ & $84(24,6 \%)$ \\
\hline
\end{tabular}

TABLA 4: Mención de requerimientos éticos en la investigación realizada en artículos de revistas indexadas en LILACS $(\mathrm{N}=23)$

\begin{tabular}{|lrr|}
\hline & Est. Farmacológicos & Est. No farmacológicos \\
Consentimiento informado & $(\mathrm{N}=84)$ & $(\mathrm{N}=107)$ \\
Privacidad de datos & $40(47,6 \%)$ & $34(31,8 \%)$ \\
Seguro efectos adversos & $2(2,4 \%)$ & $6(5,6 \%)$ \\
Revisión por Comité de Ética & $4(4,8 \%)$ & $0(0,0 \%)$ \\
Sin referencias éticas & $42(50,0 \%)$ & $58(34,6 \%)$ \\
\hline
\end{tabular}

TABLA 5: $\quad$ Referencias éticas en normas de publicación o instrucciones a los autores en revistas indexadas en MEDLINE y en LILACS

\begin{tabular}{|lrr|}
\hline & Rev. MEDLINE & Rev. LILACS \\
Revisión por Comité de Ética & $(\mathrm{N}=18)$ & $(\mathrm{N}=23)$ \\
Declaración de Helsinski & $5(27,8 \%)$ & $10(43,5 \%)$ \\
Consentimiento informado & $4(22,2 \%)$ & $7(30,4 \%)$ \\
Otros & $9(50,0 \%)$ & $3(13,0 \%)$ \\
Sin referencias éticas & $5(27,8 \%)$ & $3(13,0 \%)$ \\
\hline
\end{tabular}

Del total de ensayos clínicos con medicamentos publicados en MEDLINE y/o LILACS (177 artículos) se puede apreciar que:

- No hay estudios clínicos Fase I.

- Hay dos estudios fase II $(1,1 \%)$. En ambos se cumplen todos los requisitos éticos exigidos para investigación en seres humanos.
- Hay 44 estudios fase III (24,9\%). En ellos se encuentra referencias a cumplimiento de algunos de los requisitos éticos exigidos en un $81 \%$.

- Hay 131 estudios fase IV (74,0\%). En ellos se encuentra referencias a cumplimiento de algunos de los requisitos éticos exigidos en un $33 \%$. 
Evaluación bioética de trabajos de investigación en seres humanos publicados en América Latina y el Caribe - R. Mancini y F. Lolas

\section{Comentario}

Debido a que el análisis de la política editorial en materia de bioética se hizo sobre la base de revisar las normas editoriales o las instrucciones a los autores, sin disponer de una información directa entregada por los editores (excepto en ocho casos), podría existir alguna información parcial u omisión al respecto. En todo caso, por tratarse de instrucciones que muchas veces constituyen el único vínculo entre los investigadores y los editores, adquieren una gran importancia para evaluar la relevancia que le asignan al cumplimiento de estándares éticos en el estudio clínico $(4,5)$.

La exigencia de cumplir una o más de las directrices éticas principales para la investigación en seres humanos se encuentra en 22 de las 41 revistas analizadas, sin que se aprecie una diferencia significativa entre las indizadas en MEDLINE y en LILACS. Es preocupante que tratándose de revistas científicas que específicamente se dedican a publicar estudios clínicos en seres humanos, haya un $46,3 \%$ de ellas en que no hace ninguna mención a la necesidad de cumplir con normativas éticas básicas en las investigaciones enviadas para divulgación. No obstante, al menos en dos revistas que no consignan esa información en las instrucciones a los autores se pudo detectar (por la encuesta enviada a los editores) que existe una revisión en ese sentido por parte de uno o más miembros del Comité Editorial.

La mayor preocupación que demuestran los editores se refiere al consentimiento informado en las revistas indizadas en MEDLINE (50\%) y a la aprobación por comité de ética en las revistas registradas en LILACS (43\%). Aunque el grupo de revistas es reducido como para generalizar una opinión, se podría pensar que el criterio que regula MEDLINE da mayor relevancia a la autonomía del sujeto, a través del consentimiento informado (orientación más bien anglosajona); en cambio, el perfil encontra- do mayoritariamente en las revistas latinoamericanas indexadas en LILACS se inclina principalmente hacia el principio de la beneficencia, a través de la protección de los derechos de las personas que brinda el comité de ética (modelo paternalista).

Al revisar los aspectos éticos que son mencionados en el texto de los artículos se puede notar que existe una mayor preocupación por parte de los investigadores de dejar constancia que se obtuvo el consentimiento informado de los sujetos y la aprobación del comité de ética correspondiente cuando se trata de ensayos con medicamentos u otros productos biológicos, respecto de otros tipos de estudios clínicos. En efecto, en el primer caso -considerando ambos grupos de revistas- se señala la obtención de consentimiento informado en 87 de 177 ensayos (49\%) y la aprobación por comité de ética en 81 de 177 estudios (46\%). En cambio, cuando se trata de estudios clínicos que no involucran fármacos, se señala obtener el consentimiento informado sólo en el $32 \%$ de las investigaciones (143 de 448) y la revisión por comité de ética en el 27\% (121 de 448). Quizás esto se explique por la mayor exigencia regulatoria que se hace a nivel de la industria farmacéutica internacional en el primer tipo de investigaciones, lo que no ocurre al no haber entidades controladoras en el ámbito local para los otros tipos de estudios ( 6 ).

Por otra parte, hay un mayor cumplimiento de disposiciones éticas en los ensayos clínicos de fase II o III que en los de fase IV, lo que coincide con las mayores exigencias internacionales que se hace a los primeros, por tratarse de fármacos aún no aprobados para su uso en los países donde se hace la investigación.

Otros requisitos éticos revisados en el texto de los artículos no tuvieron relevancia cuantitativa, aunque vale mencionar que la protección ante efectos adversos explicitada o cumplida por los investigadores ante su aparición, se observa en cerca del $7 \%$ de las 
investigaciones con fármacos o productos biológicos y apenas es mencionada en los restantes estudios, incluso considerando que todos ellos implicaron procedimientos o técnicas que eventualmente podían producir efectos deletéreos en los sujetos.

Aunque podría considerarse que la consignación de revisión y aprobación por un comité de ética independiente implicaría el cumplimiento de esas disposiciones éticas en el protocolo de estudio, no es suficiente salvaguarda de los derechos de las personas ante eventos adversos, ya que en la gran mayoría de los países no existen sistemas de seguros que operen automáticamente en estos casos y los comités de ética no disponen de atribuciones legales como para obligar a los investigadores a cumplir esa obligación. Por lo tanto, que no se señale especialmente la forma en que se atenderán los efectos adversos es un grave problema pendiente de solución ( 7 ).

En general, fuera de las diferencias señaladas y que no son relevantes para efectos de esta investigación, no hubo una disimilitud significativa entre ambos grupos de revistas, respecto del cumplimiento de estándares éticos en la investigación. Esto permite sugerir, ya que el número de revistas y artículos es reducido como para hacer afirmaciones definitivas, que la indización en la base de datos MIDLINE no considera de forma especial el cumplimiento explícito de requisitos éticos en las investigaciones publicadas, como norma de calidad de las revistas científicas. Más clara aún es esta constatación si se considera que la base de datos LILACS se autodefine como "total" sin plantear criterios de exclusión especiales- y se obtiene por resultado un cumplimiento muy similar de requisitos éticos.

Como comentario en lo general, cabe mencionar la dificultad que existió para obtener los textos completos de las revistas, a pesar de todos los métodos utilizados (BIREME, solicitud de préstamo interbibliotecario, solicitud directa a los edi- tores). La imposibilidad de obtener los artículos en un gran número de publicaciones (10 de las revistas indexadas en MEDLINE y 151 de las indexadas en LILACS) permite suponer que, aunque quizás estén accesibles en el medio local, no lo están para el lector científico internacional -razón de ser de las bases de datos revisadas- cumpliendo dichas revistas, por lo tanto, muy parcialmente su objetivo de difusión científica $(8,9)$.

\section{Conclusiones}

1. Existe una gran cantidad de publicaciones en las revistas más difundidas de los países que efectúan el mayor número de investigaciones en seres humanos en la Región de América Latina y el Caribe, que no explicitan el cumplimiento de requisitos éticos básicos en dichas investigaciones. Aunque en un cierto porcentaje podría asumirse que la revisión y aprobación por un comité de ética da cuenta implícitamente de ese cumplimiento, persiste un número considerable de estudios que no tienen ningún respaldo ético manifiesto.

2. Las normas de publicación e instrucciones a los autores -corroborada en algunos casos por encuesta a los editores- dan cuenta de una preocupación por el cumplimiento de normativas éticas en poco más del $50 \%$ de las revistas. Esto es de especial preocupación, ya que se trata específicamente de revistas que publican investigaciones en seres humanos.

3. La información obtenida, complementado estudios anteriores, permite disponer una aproximación importante a la situación real en que se encuentra la ética de la investigación en seres humanos en América Latina y el Caribe. Su divulgación a los países de la Región debería servir de incentivo para la creación de comités de evaluación bioética en aquellos lugares e instituciones que no cuenten con ellos. También es de esperar que se despierte la inquietud de los encargados de evaluar los trabajos de inves- 
Evaluación bioética de trabajos de investigación en seres humanos publicados en América Latina y el Caribe - R. Mancini y F. Lolas

tigación que son propuestos para su publicación en las revistas científicas de prestigio de la Región, en cuanto a la revisión ética de dichas publicaciones

\section{Palabras finales}

Esta clara confirmación de la necesidad imperiosa de disponer de mecanismos regulatorios y de control bioético de las investigaciones en seres humanos en la Región de América Latina y el Caribe debe ser vista de manera positiva. Algunos investigadores plantean que la regulación ética puede crear barreras infranqueables para la creatividad y el desarrollo de la ciencia. Esto podría ser efectivo si ese control es efectuado por personas mal preparadas y a través de procedimientos burocráticos ineficientes.

Sin embargo, normativas claras y compartidas para la investigación clínica, comités de evaluación ético-científicos con integrantes capacitados y criterios bioéticos bien definidos en las políticas editoriales, deberían permitir una regulación ética expedita $\mathrm{e}$ indispensable para proteger los derechos de las personas y ayudar a los investigadores a hacer una ciencia de mayor calidad, precisamente por esa preocupación por los sujetos humanos que son la razón de su quehacer.

\section{ANEXO 1}

\section{Grupo A del estudio}

Revistas Latinoamericanas indexadas en MEDLINE y LILACS sobre investigación en seres humanos posibles de obtener en texto completo

\section{ARGENTINA:}

1. Acta Gastroenterológica Latinoamericana (Buenos Aires)

2. Acta Physiologica, Pharmacologica et Therapeutica Latinoamericana (Buenos Aires)

3. Medicina (Buenos Aires)

4. Revista de la Facultad de Ciencias
Médicas, Universidad Nacional de Córdoba BRASIL:

5. Arquivos Brasileiros de Cardiologia

6. Cadernos de Saúde Pública

7. Revista Associação Médica Brasileira (Sao Paulo)

8. Revista do Hospital Das Clínicas (São Paulo)

9. Revista do Instituto de Medicina Tropical de São Paulo

\section{CHILE:}

10.Revista Médica de Chile

11. Boletín Chileno de Parasitología

CUBA:

12.Revista Cubana de Medicina Tropical

MEXICO:

13. Gaceta Médica de México

14.Archives of Medical Research (México)

15. Archivos del Instituto de Cardiología de México

16.Ginecología y Obstetricia de México (México)

\section{PUERTO RICO:}

17.Puerto Rico Health Sciences Journal VENEZUELA:

18.Investigación Clínica (Maracaibo)

\section{ANEXO 2}

\section{Grupo B del estudio}

Revistas Latinoamericanas indexadas en LILACS (y no en MEDLINE) sobre investigación en seres humanos posibles de obtener en texto completo

\section{ARGENTINA:}

1. Medicina Intensiva (Buenos Aires

2. Acta Psiquiátrica y Psicológica de América Latina (Buenos Aires)

3. Revista Argentina de Cancerología (Buenos Aires) 
4. Obstetricia y Ginecología Latinoamericana

5. Archivos Argentinos de Pediatría

\section{BRASIL:}

6. Brazilian Journal of Medical and Biological Research

7. Memórias do Instituto Oswaldo Cruz

8. São Paulo Medical Journal

9. Acta Cirúrgica Brasileira

10.Arquivos de Neuro-Psiquiatria

11.Revista Brasileira de Cirugia Cardiovascular

12.Revista da Sociedad Brasileira de Medicina Tropical

\section{CHILE:}

13. Revista Chilena de Pediatría

14. Revista Chilena de Neuro-Psiquiatría

15.Reumatología (Santiago de Chile)

16.Revista Chilena de Infectología

\section{CUBA:}

17.Revista Cubana de Investigación Biomédica

18. Revista Cubana de Medicina

\section{MEXICO:}

19.Salud mental (México)

20.Enfermedades Infecciosas y Microbiología (México)

21. Revista de la Asociación de Medicina Crítica y Terapia Intensiva (México)

22.Revista Mexicana de Anestesiología

\section{PUERTO RICO:}

* Boletín de la Asociación Médica de Puerto Rico. Es la única indexada en LILACS, pero sin artículos en los años correspondientes a la revisión $(1997,1998)$ por lo que se excluyó del estudio

\section{VENEZUELA:}

23. Gaceta Médica de Caracas (Caracas)

\section{Referencias}

1. Mancini R, Lolas F. El Programa Regional de Bioética y la investigación en salud. XXXIV Reunión del Comité Asesor de Investigaciones en Salud de la OPS/OMS, 12-14 de julio de 1999, Washington D.C. [On Line] Available from: URL: http://www.PAHO/ OPS/ACHR

2. Figueroa P. Aspectos éticos de la investigación biomédica en Iberoamérica y el Caribe. Documento de trabajo: Georgetown University, Kennedy Institute of Ethics, Washington, DC: Kennedy Institute of Ethics; 1994.

3. CONACYT México. Informe sobre la situación de la investigación en Latinoamérica y el Caribe. [On Line] Available from: URL: http://www.siicyt.com.mx/.

4. Sandenberg T, Muller S, Pereira H, Oliveira R, Hossne W. Análisis de los aspectos éticos de la investigación en seres humanos contenidos en las Instrucciones a los Autores de 139 revistas científicas brasileñas. Acta Bioethica 2000; 6(2): 293-307.

5. Malacara JM. La evaluación de la investigación médica. Revista Investigación Clínica 1997; 49(4): 303-8.

6. Macklin R. Investigación colaborativa internacional: acontecimientos recientes. En: Pellegrini A, Macklin R, (eds). Investigaciones en sujetos humanos: experiencia internacional. Serie Publicaciones Programa Regional de Bioética OPS/OMS, Santiago de Chile: Universitaria; 1997, p. 47-61.

7. Emanuel E. ¿Qué hace que la investigación sea ética? Siete requisitos básicos. En: Pellegrini A, Macklin R, (eds). Investigaciones en sujetos humanos: experiencia internacional. Serie Publicaciones Programa Regional de Bioética OPS/OMS, Santiago de Chile: Universitaria; 1997. p. 33-46.

8. Pearn J. Publication: an ethical imperative. British Medical Journal 1995; 310:1313-15.

9. Lolas F. Ética de la publicación médica: legalidad y legitimidad. Acta Bioethica 2000; 6(2): 283-91. 